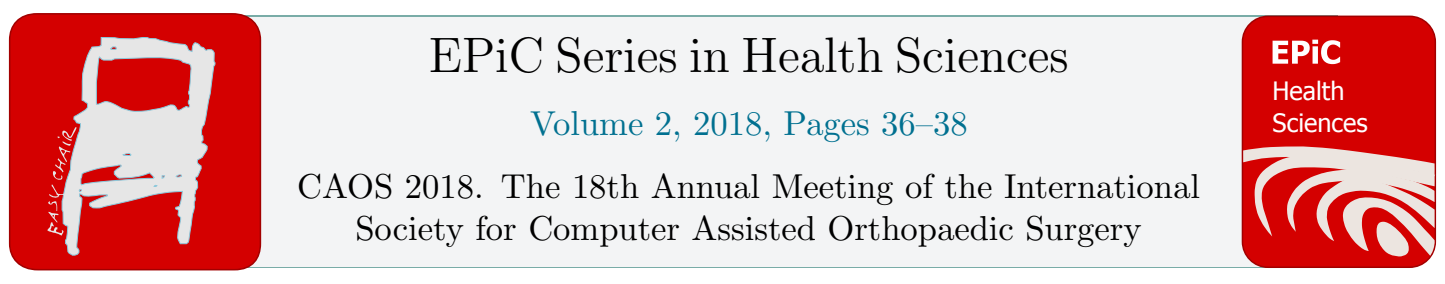

\title{
Accuracy and reliability of full-length lower limb radiographs in the measurement of coronal alignment
}

\author{
Yifei Dai ${ }^{1 *}$, Matthew Peterson ${ }^{1}$, Katharine Wurm ${ }^{2}$ \\ ${ }^{1 *}$ Exactech Inc, Gainesville, FL, 32653, USA \\ ${ }^{2}$ Medical Metrics Inc, Houston, Texas, USA \\ yifei.dai@exac.com
}

\begin{abstract}
This study investigated the accuracy and reliability of using full-length lower limb radiographs to measure alignment outcomes of computer-assisted total knee arthroplasty. Simulated radiographs were created from cadaveric whole leg CT scans. Three analysts performed repeated measurement of the hip-knee-ankle (HKA) angle, blinded to the ID of the subject and image repetition. In addition, simulated radiographs with internal/external- limb rotation were measured to assess sensitivity of the measurements. The result demonstrated high accuracy and reliability of the measurement, with low sensitivity to limb rotation. The findings supported the use of full-length lower limb radiographs to measure clinical outcomes in HKA.
\end{abstract}

\section{Introduction}

As computer-assisted (CA) total knee arthroplasty (TKA) has been shown to offer improved accuracy in postoperative alignment, weight-bearing full-length lower limb radiographs were used as the gold standard for the alignment measurement [1,2]. To properly demonstrate the alignment accuracy in CA TKA, the error in the measurement tool itself should not significantly confound the results. As intraoperatively measured resection error from CA TKA has been reported to be sub-millimeter [3], the clinical outcome of the CA TKA alignment should aim for degree level accuracy. In addition, although most hospital have protocol in place to control limb rotation during radiographing process, some degrees of rotation cannot be entirely avoided. Ideally, the measurement of alignment should be robust to limb rotation that may causes oblique anteroposterior (AP) view during radiography.

The purpose of this study was to assess the accuracy and reliability of full-length lower limb radiographs in the measurement of alignment outcome of CA TKA and the sensitivity of the measurement to limb rotation. 
Accuracy and reliability of full-length lower limb radiographs in the measurement of ... Y. Dai et al.

\section{Materials and Methods}

CT scans of 3 cadaveric legs (pelvis to foot) in full extension were segmented to surface models, followed by virtual TKA (3-matic research, Materialise NV, Leuven, Belgium). The "true" postoperative hip-knee-ankle (HKA) angle was determined as the coronal angle formed by: 1) tibial mechanical axis: the line connecting the mediolateral (ML) centers of the talus and the tibial component; and 2) femoral mechanical axis: the line connecting the spherical center of the femoral head and the ML center of the femoral component notch.

Simulated full-length coronal radiographs were generated from the CT data at the default AP view (dictated by the original CT scanning position), and at $5^{\circ}, 10^{\circ}, 15^{\circ}$ of internal and external limb rotation. Using the corresponding HKA definition in 2D, 3 analysts each evaluated the simulated radiographs at the default view 3 times. Next, the 3 analysts each evaluated the entire set of the rotated radiographs. The analysts were blinded to the repeated and rotated radiographs.

Accuracy of the radiographic alignment measurement was calculated referencing the "true" HKA in the default view. The impact of limb rotation was quantified by the change in HKA measured at varying internal/external rotation angles relative to the default view. The inter- and intra- analyst reliability of the measurements were assessed by Intraclass Correlation Coefficient (ICC).

\section{Results}

HKA measurements based on full-length radiographs produced errors of sub-degree level and demonstrated strong inter- and intra- analyst reliability (Table 1). Rotation of the limb up to $15^{\circ}$ had only sub-degree impact on HKA (Table 2). Radiographs with rotated limb exhibited high inter-analyst reliability for the HKA measurement (ICC $=0.992)$.

\begin{tabular}{lcc}
\hline Accuracy & Signed* & Unsigned \\
Error in HKA $\left(^{\circ}\right)$ & $-0.6 \pm 0.5$ & $0.6 \pm 0.4$ \\
\hline Reliability & ICC & $95 \%$ CI \\
Inter-Analyst & 0.984 & {$[0.727,1.000]$} \\
Intra-Analyst & & \\
Analyst 1 & 0.981 & {$[0.630,1.000]$} \\
Analyst 2 & 0.980 & {$[0.760,0.999]$} \\
Analyst 3 & 0.998 & {$[0.977,1.000]$} \\
\hline * Negative value indicates the radiograhic measurement on \\
HKA is more varus compared to the "true" HKA.
\end{tabular}

Table 1. Accuracy and reliability of the HKA measured based on full-length radiographs at the ideal view (perfect AP limb position during radiography). 
Accuracy and reliability of full-length lower limb radiographs in the measurement of ... Y. Dai et al.

\begin{tabular}{lccc}
\hline $\begin{array}{l}\text { Error in HKA } \\
\mathbf{(}^{\circ}, \text { Unsigned, Mean } \pm \text { Standard Deviation) }\end{array}$ & $\mathbf{5}^{\circ}$ Rotation & $10^{\circ}$ Rotation & $\mathbf{1 5}^{\circ}$ Rotation \\
Internal and External Rotation Combined & $0.6 \pm 0.5$ & $0.5 \pm 0.5$ & $0.6 \pm 0.7$ \\
Internal Rotation & $0.6 \pm 0.6$ & $0.7 \pm 0.6$ & $0.8 \pm 0.8$ \\
External Rotation & $0.5 \pm 0.5$ & $0.4 \pm 0.5$ & $0.4 \pm 0.3$ \\
& ICC & $95 \% \mathrm{Cl}$ & \\
Reliability & 0.992 & {$[0.978,0.997]$} & \\
Inter-Analyst & &
\end{tabular}

Table 2. Impact of limb rotation on the accuracy of HKA measurement. Inter-analyst reliability of the measurement on the rotated radiographs is also presented.

\section{Discussion}

The data demonstrated the high accuracy of using full-length lower limb radiographs for the measurement of postoperative coronal alignment outcome. The average error and standard deviation found in this study were both lower than those from CA TKA [1,2]. In addition, the measurement did not exhibit sensitivity to limb rotation for up to $15^{\circ}$. Furthermore, strong inter- and intra- analyst reliability was found, confirming the robustness of the measurement method. In conclusion, full-length lower limb radiograph is an accurate and reliable imaging modality for the quantification of HKA in the clinical studies for CA TKA.

\section{References}

[1] Blakeney WG, Khan RJ, Wall SJ. Computer-assisted techniques vs conventional guides for component alignment in total knee arthroplasty: a randomized controlled trial. J Bone Joint Surg Am. 2011;93:1377-84.

[2] Chen JY, Chin PL, Tay DK, et al. Less outliers in pinless navigation compared with conventional surgery in total knee arthroplasty. Knee Surg Sports Traumatol Arthrosc 2014 ; 22 : 1827-32.

[3] Ensini A, Catani F, Leardini A, et al. Alignments and clinical results in conventional and navigated total knee arthroplasty. Clin Orthop Relat Res. 2007;457:156-62. 